\title{
Moroccan TV Broadcasters and Viewership Changes in the Digital Age: An Exploratory Study
}

\author{
A. Dadouh and A. Aomari
}

\section{ABSTRACT}

\begin{abstract}
In the first two decades of the $21^{\text {st }}$ century, the relationship between viewers and television underwent major changes, with the advent of technologies that led to new viewing habits. This situation starts now to question the ability of broadcasters to build and retain loyal viewers. As a response, broadcasters are adapting their contents and distribution to fit in the new digital world. While studying the possible impacts of the efforts made by broadcasters, we complete our analysis by addressing also the factors that guide consumer choices through media use models, which focus on the psychological, emotional, personal and environmental aspects of media consumption choices. Through interviews with Moroccan broadcasters, this paper aims to identify which behavioral aspects and innovation levers broadcasters should take into account to build audience loyalty in the era of digital media.
\end{abstract}

Keywords: Television, broadcasters, viewing behavior, multiplatform, loyalty.

\section{INTRODUCTION}

With the popularization of internet access, the multiplicity of online platforms (social networks, video on demand, etc.) and the variety of electronic devices (smartphones, tablets, etc.), Crawford [1] discusses the migration of traditional television viewers to new program providers who are described as "customer friendly". To highlight these changes, Perticoz \& Dessinges [2] suggest three trends to conceptualize the questions related to the current modes of audio-visual consumption, by evoking the example of the consumption of television series. These trends are viewing autonomy regarding schedules, connected interfaces to watch programs and online participation.

In this situation of technological media convergence, broadcasters have adopted multi-platform strategies to deliver their content to a wider audience. Leaver [3] explains that a multi-platform approach can be either a duplication of existing content on additional digital platforms, an adaptation of existing production to different platforms, or even the creation of additional layers of content that are mainly webbased.

In the world of media, researchers rely also on peculiarities closely linked to different personal, emotional and environmental variables that lead to selective exposure. Krcmar et al. [4] use this approach as it recognizes that our choices are likely guided by affective, psychological, and functional factors; and seeks to uncover what those factors are and the interrelations between these factors and our ultimate media choices. Therefore, we could not neglect the importance of those factors in our analysis.

Our literature review sheds light on innovation and multiplatform strategies as a response by broadcasters to the challenges of their changing environment. Nevertheless, we note that previous works remain insufficient to establish links between these strategies and the concept of loyalty, and between media use models and the current television context Thus, broadcasters' perceptions of the factors driving media use are important to complete the analysis. The focus of our investigation is therefore on the main issues of the digital era for broadcasters and on the adoption of innovation to build viewer loyalty, particularly by implementing multiplatform strategies.

To sum up, the purpose of this paper is to identify which behavioral trends and innovation levers broadcasters would consider in order to build audience loyalty in the era of digital media. We will try to achieve our purpose through a literature review and a field qualitative study that targets Moroccan broadcasters.

\section{LITERATURE REVIEW}

The widespread internet access and the variety of online platforms (social media, streaming services, etc.) and digital devices (smartphones, tablets, etc.) have imposed a new reality for television broadcasters. Thus, this work focuses more on the new behaviors of video consumption and on broadcasters' multiplatform strategies as a natural response to this context.

\section{A. Video Consumption Habits in the Digital Age}

In the past two decades, the way people consume video contents received much attention from scholars. One of the most interesting descriptions of how television is changing comes from Medina et al. [5] who stress that television is changing on several levels. Particularly, it is transitioning 
from analogue to digital, from scheduled broadcasts to ondemand TV, from lean back (passive) to lean-forward (active) media, from straight watching to consumption of content connected to additional services, from the solitary TV viewer to the viewer who is part of social networks and communities related to TV content, from single-screening to multiscreening, etc.

From these trends, we can retain three essential elements for our analysis: viewing autonomy, connected devices and online participation.

\section{Viewing Autonomy}

When researchers speak about an original way of watching television, "appointment viewing" is one of the most relevant features. As explained by Hecht [6], appointment viewing occurs when viewers schedule time - or make appointments - to watch their favorite shows, while Perticoz \& Dessinges [2] consider that autonomy only becomes possible after the moment of the first broadcast. This autonomy has always known a spectacular evolution since the 1970s with the appearance of the video recorder and then the succession of different technologies thanks to satellites and the Internet to weaken the grip of appointment viewing.

Since the arrival of satellite television during the 1990s, more and more countries have been witnessing a liberalization of the media landscape, leading to a spectacular surge of television empires. This has given viewers, a wide range of options in terms of programs as well as viewing times with channels airing their programs 24 hours a day, and being able to rerun programs out of prime time.

In the 2000s, the public saw the emergence of social networking platforms including YouTube for sharing video content, but also the rise of video-on-demand platforms such as Netflix that came with content available online at any time. This would be considered as a huge step forward, giving more autonomy to the viewer, reaching the form that Perticoz and Dessinges [2] describe as self-programming. By this term, they mean "a way of consumption that is desynchronized visà-vis the program grid, potentially fragmented on different media according to different times and spaces. In any case, it is a programming that adjusts to the time, the media and the desires of consumers".

Thanks to this autonomy, new sorts of behaviors are now in trend, notably Binge watching, which can be defined as "the experience of watching multiple episodes of a program in one sitting" [7]. The video-on-demand industry has used binge watching as a marketing tool, exploiting this form of serial reception as a unique selling point [8]-[10].

\section{Connected Devices}

In a context marked by a variety of connected devices, on which one can watch video contents anywhere, the viewing process is more likely to be extended to other devices while the "Media Multitasking" emerges. Bardhi et al. [11] define this concept as the practice of simultaneously participating in multiple exposures to multiple forms of media.

Nevertheless, Nee \& Dozier [12] argue that consumers are using multi-screen in a way that is unrelated to the content on TV. In support of this, they points to previous studies showing that consumers are using email, social media and the Internet as forms of second screen activity. Yet, while media multitasking might be a threat to viewers' attention, Dadouh \& Aomari [13] highlight the fact that it could also be an opportunity for content makers to enrich the experience of video consumption through "Second Screening", especially for broadcasters to build an interactive TV experience. In this purpose, we identify two main behaviors that are deemed to be of interest to study when it comes to media multitasking: the parallel use of two or more digital devices while watching television [14]; and the use of a second device to complement what is being watched on TV, particularly at major live events [15].

As information technologies have increasingly challenged the traditional way of consuming audiovisual content, by enabling viewers to consume it anywhere and anytime, SeeTo et al. [16] link this also to the ever-growing number of different portable devices like smartphones and tablets. The trend is particularly due to low cost of Internet access, combined with advanced hardware capabilities. Together, they have made it possible for the public to download and view videos on mobile devices [16].

\section{Online Participation}

Social media have created a space for discussion around video contents generally and TV programs specifically, as they did for other goods or services marketed by brands in different industries.

While watching television, viewers increasingly use a second screen such as their laptop or smartphone to communicate with others, for example by expressing their opinion on the content of a specific program or by reading comments written by friends or unknown peers [17].

Guo's study [18] identifies four underlying dimensions of social engagement behavior with television programs: vertical involvement, diagonal interaction, horizontal intimacy and horizontal influence.

- Vertical involvement: it represents the lowest degree of this social viewing model, reflecting the participatory behavior related to the main content and/or ancillary information of a program. It is therefore a variety of points of contact via social media;

- Diagonal interaction: it represents the dialogues between the audience and media figures through the Twitter microblog;

- Horizontal intimacy: it goes beyond diagonal interaction to characterize the audience's affection and feeling for television programs, measuring peer-to-peer activities such as expressing one's own opinions and responding to the views of others in blogs and online forums;

- Horizontal Influence: goes beyond the feeling itself to mark the personal and social identification of an individual audience; and also represents the potential for the individual audience to influence their friends in social networks by sharing and recommending the program.

\section{B. Innovation and Multiplatform Strategies of TV Broadcasters}

Innovation is a fact of life for the media industry, and it was ever thus. Along their history, media organizations have always needed to innovate - as indeed have organizations in other industries. In recent decades, however the requirement for innovation in the media industry has become both more urgent and more challenging with the increase in the pace and scope of technological evolutions [19].

Since the beginning of the 20th century, broadcasters have 
shown great adaptability to technological developments, even taking advantage of them to develop their offer and remain at the forefront of the media landscape [13]. Accordingly, Medina, et al. [5] find that broadcasters are trying to develop sophisticated tools for integrating connected television. Their aim is to drive viewer integration, engagement and loyalty in order to increase popularity, online audiences and advertising revenues. In addition, television applications and multiscreen solutions provide new ways of use.

A multi-platform distribution approach can be either a duplication of existing content on additional digital platforms (e.g. by providing linear TV content online via live streaming), or an adaptation of existing production to different platforms (e.g. by re-editing it) or by adding additional layers of content. The creation of additional original content (e.g. web-based) and other ancillary materials to complement existing linear offerings can be at the heart of multi-platform distribution strategies [3].

Baumann \& Hasenpusch [20] argue that a clear notion of concepts and definitions within such an emerging field is necessary to identify and develop new opportunities for value creation. Thus, they suggest defining and gathering the terms into two groups: hybrid television and multi-screening.

\section{Hybrid TV}

Hybrid-TV mainly includes concepts related to technology and devices. It encompasses IPTV (Internet Protocol Television), HbbTV (Hybrid Broadcast Broadband) and Over-The-Top TV (OTT-TV) with its subcategories. The main difference between hybrid televisions is the network structure that allows consumers to receive linear contents and access contents on demand. IPTV allows the broadcasting of audiovisual content over a managed and closed network that provides better quality control. As for OTT-TV, it allows content to be broadcast without an information provider controlling the signals. This hybrid character is more evident with HbbTV, which is a developed combination of satellite (Broadcast) and high speed Internet (Broadband) on one screen.

Despite the technical differences between OTT-TV and IPTV, they both enhance the viewing experiences of audiovisual content consumption through video on demand (VOD), catch-up TV, but also live broadcasting (Live Streaming). In the MENA region, MBC Group's "Shahid" is one of the most prominent examples for a service gathering both video on demand and live streaming. In Morocco as well, public broadcasters like SNRT and SOREAD 2M also have their online streaming and catch-up TV services.

As for the HbbTV system, García-Avilés [21] believes that its gradual implementation, with a technology based on the HTML standard, allows the integration of the TV set with web access, thus offering a more interactive TV experience. As far as the adoption of this technology is concerned, the European zone is a pioneer. In the MENA region, the first HbbTV experience is the result of a partnership in 2014 between the technology provider, Selevision, and the satellite operator Arabsat. The service is however available on a limited number of TV channels.

\section{Multi-screening}

Multi-screening refers to terms and concepts related to consumer behavior. It includes "Second Screening", "Dual Screening" as well as "Social TV". The work of Baumann and
Hasenpusch [20] makes a distinction between the terms "Second Screening" for activities related to broadcast content and "Dual Screening" for independent activities. It is also a good idea for broadcasters to pay special attention to social media discussions related to linear television content. This combination of audiovisual content and social networks, called "Social TV", further promotes a two-way communication flow.

Fleury et al. [22] believe that it is interesting to study how to successfully combine television and mobile technologies in a cross-media or second-screen environment, and that it is fundamental to determine the types of interactions that television consumers would like to use via a second screen application and how it should be designed.

On social media, broadcasters offer many interactive activities around their programs: previews and teasers prior to broadcast, games, voting, questions to guests, but also viewers' opinions during and after the program's broadcast. Mawenzi Partners' report [23] suggests three benefits for audiovisual groups that are part of this trend:

- Before broadcasting: creating and maintaining the buzz.

- During broadcasting: organizing and developing the social audience.

- After broadcasting: capitalizing on brand awareness and loyalty building.

\section{Viewer Loyalty from a Media Use Perspective}

The issue of viewer loyalty has received some attention from researchers, but remains of vital interest to broadcasters. Sabavala and Morrison [24] propose loyalty as a dimension that programming decision-makers could benefit from in program ratings. Thus, loyalty towards broadcasters would be defined as "the extent to which viewers tend to watch a channel's programs rather than divide their viewing time equally among the different channels" [25].

By analyzing the literature on the concept of viewer loyalty, we can already notice a convergence with the different approaches to loyalty addressed by marketing research with aspects related to cognitive and affective factors, especially in terms of satisfaction, trust and commitment. In order to better deepen the understanding of viewer loyalty, these points of convergence could be of scientific interest, within the framework of a reflection that also takes into consideration more media use models related to personality and socio-demographics.

\section{Sought Objectives}

Generally, people consume media contents for some reasons: they are seeking to achieve objectives. This phenomenon is better discussed through uses and gratifications theory. Gratification remains a perception of the satisfaction of public needs through media consumption [26]. Katz, Haas and Gurevitch [27] summarize these needs as cognitive needs, affective needs, personal integration needs, social integration needs and needs to release tensions. If, in a general framework, satisfaction is based on the gap between product expectations and performance, in the television context, it would be based on the gap between the expectations formed with regard to television content before exposure (gratifications sought) and the satisfaction obtained subsequently through its consumption (gratifications 
obtained).

\section{Personality Factors}

Finn's study [28] can be considered among the first to have tried to establish a link between personality and media use, by analyzing the relationship that could exist between the five fundamental personality traits, namely extroversion, neuroticism, openness to experience, agreeableness and conscientiousness, on the one hand, and media exposure on the other. From this work, Finn [28] draws the conclusion that personality traits could at least predict which type of media is used. Weaver [29] also shares this point of view. He argues that horror films attract individuals with a high degree of psychoticism (characterized as impulsive and nonconformist). Those who are more neurotic show a preference for news programs.

\section{Emotional Factors}

The way in which individuals evaluate, affiliate and actively interact with characters in media is an aspect on which explanations of pleasure in media consumption focus. Among the first explanations, we find the disposition theory that was first proposed in the work of Zillmann and Cantor [30]. In fact, disposition approaches can be applied to enjoyment of a wide range of content and differ somewhat from one approach to the next, depending on the content under consideration [31].

\section{Mood Factors}

Individuals strive to minimize negative mood states and maximize or maintain positive mood states by arranging their environment (including media) to achieve that goal [4]. People choose a medium that produces a physiological arousal contrary to the one that is felt, leaving them better off than before. In other words, when we are more excited, we tend to consume more calming contents. Otherwise, when we are bored, we tend to consume exciting contents. Moreover, when we feel bad, we avoid exposition to contents that remind us of the origin of that feeling.

However, some works such as Knobloch [32] introduce states in which individuals prefer to maintain mood states rather than ending them. This process is called "Mood adjustment", in which Knobloch [32] suggests that media selection and use may be one way we maintain or adjust our mood in anticipation of a situation requiring that mood.

\section{Sociological Factors}

Researchers generally identify sociological-level variables as social controls guiding media consumption. For example, the works of Strasburger, Wilson \& Jordan [33] and Krcmar et al. [4] discuss media consumption in relation to the gender variable. While the former finds that, among children, boys watch more television and play more video games than girls, the latter suggest that among adults, women consume slightly more television than men. Papacharissi and Rubin [34] and Saxena [35] focus on the role of quality of life and material conditions. As far as the age variable is concerned, it is covered by Gauntlett and Hill [36] and Bondad-Brown et al. [37], focusing on the differences that might exist between the different generations: silent (+65 year olds), "Baby boomers" (50-64 year olds), Generation X (35-49 year olds), Generation Y (21-34 year olds) and Generation Z (15-20 year olds).

\section{EMPIRICAL STUDY}

We conducted a field qualitative study targeting Moroccan broadcasters to enrich the theoretical framework with more practical data from the Moroccan market. Therefore, we had interviews with managers from marketing, digital and scheduling departments in five Moroccan media groups: SNRT, SOREAD 2M, Medi 1, MAP and Hit Radio (a radio group expanding to TV broadcasting).

\section{A. Method}

For the constitution of our sample, we opted for a multiple case design, through conducting interviews, while taking into account the criteria of diversification and saturation, which are important in this chosen process.

As our method considers saturation as a main component of our sampling, we stopped after eight interviews. The data gathered showed that conducting more interviews could no longer be enriching, as the main items in the interviewees' answers had started to be repetitive.

We opted for a semi-structured, open-ended interview, so that we could introduce new ideas during the interview based on what the interviewee says, while respecting the general framework of the addressed topics. Our choice is justified on the one hand by the precision it allows in terms of discussed topics [38]; and on the other hand, by its flexibility and the speed of analysis it allows at a lower cost [39].

As a companion while conducting the interviews, the interview guide allowed us to list the themes and questions to discuss and even enter the responses as the interview progresses. It consisted of three sections covering the main themes of our study. The first was devoted to audiovisual consumption behaviors and new habits in the digital age. The second section focused on the challenges of television in the digital environment. By evoking the complexities of the current situation for broadcasters, this section facilitated the transition to the third one, which was dedicated to the ways they deal with these changes. This last section explored the mindset of innovation in the corporate cultures of these organizations and discussed the role of multi-platform strategies in building viewer loyalty.

To start the analysis of the qualitative study, we first conducted a content analysis. This technique allows for the methodical, systematic, objective examination of the content of certain texts in order to classify and interpret their constituent elements, which are not totally accessible to naive reading [40]. The results analysis was made through the textual analysis software Nvivo 12 . We processed the study data by codifying the raw results of the major themes under different nodes belonging to the three main axes (new audiovisual consumption behaviors; television and changes in audiovisual consumption behavior; and innovation and multiplatform strategies).

\section{B. Results and Discussion}

We structured the interpretation of the results around three sub-topics that highlight the current audiovisual consumption behaviors, the position of the medium television amid these new digital developments and the response of broadcasters with their multiplatform strategies. 


\section{Current Audiovisual Consumption Behaviors}

Trends in video consumption can be addressed in terms of viewing times, use of different devices and participation on social media, in addition to factors that motivate viewers to consume broadcasters' contents and the objectives sought by viewers through this act of consumption. The study shows us that broadcasters are aware of the following trends in consumption habits:

- Autonomy regarding traditional TV schedules with the possibility for the viewer to organize his own viewing schedules in ways that suit him better;

- Consumption of content on different devices with different screen formats, especially on mobile devices, making it possible to watch content anywhere, to use more than one device at once and to benefit from complementarity between these devices;

- Viewers being part of the online discussion, particularly on social networks, with different forms of participation in a two-way communication with the broadcasters: requests, comments, opinions, criticism, problem reporting, sharing.

All this has been made possible mainly thanks to the ease of access to the Internet and online platforms, as well as the multitude of connected devices owned by individuals. This change affects the younger age groups more.

By consuming contents broadcast by TV channels, the study shows that there are factors specific to individuals that influence their audiovisual consumption habits in this era of media convergence. These are mainly socio-demographic (age, gender, socio-professional categories) and emotional factors (affection toward shows, hosts, celebrities).

In addition to the aforementioned factors, the audience's choices are impacted by objectives that individuals seek to achieve through the act of consuming one content or another. The objectives revealed by the study are information, entertainment and sharing.

\section{Television and the Digital Atmosphere}

The data collected focus on content and adaptation of formats and distribution as major elements in the future of broadcasters. As such, these elements are levers to ensure the survival of television and to face the threats of streaming platforms and new media.

The respondents see the credibility of content and the ability to adapt as strengths of television in the face of digital media. However, credibility could also be a source of a weakness when it comes to ethical standards and editorial policies that impose more verifications before the dissemination of news, and therefore less speed compared to a large part of digital media, who do not consider these standards. With broadcasters' large structures, this comes on top of the expenses and needs for qualified human resources that could fit to the new projects, as well as the administrative constraints for public broadcasters.

\section{Innovation and Multiplatform Strategies}

All of the interviewees' responses converge on the paramount importance of innovation as a strategic choice, mainly in the product and marketing aspects. From this perspective, broadcasters are adopting multi-platform strategies that allow them to be present and visible everywhere, on different devices and platforms, in order to ensure a certain degree of proximity to viewers. Similarly, they rely on new content formats, while remaining true to the values of reliability, in order to provide engaging contents.

For the interviewees, those are the fundamentals of viewer loyalty. To this end, broadcasters are trying to adapt to different screens, especially mobile. Furthermore, they strengthen their presence on social networks through diversified and adapted contents and posts. This matches with their multi-platform strategies, and their efforts aimed at leveraging streaming platforms to reach larger audiences at any time through catch-up TV and VoD.

\section{CONCLUSION}

The variety of electronic devices and online platforms has given viewers many possibilities for the consumption of audiovisual content. By choosing to study the phenomenon from broadcasters' angle, we have tried through this work to analyze how they perceive the change and how they respond to it. These new trends were highlighted in this paper by means of a literature review and a field study.

Today, television is changing on several levels: it is transitioning from analogue to digital, from scheduled broadcasts to on-demand TV on the internet, ... from the solitary TV viewer to the viewer who is part of social networks and communities related to TV content, from single-screening to multi-screening, etc. [5]. Thus, we have tried to study the impacts of the changes in terms of viewing autonomy, device variety and online participation on broadcasters' strategies. In this regard, the changes are considered by the interviewees as opportunities for more proximity towards audiences and then for building loyalty.

Moreover, evidence from this study showed that among different media use models, sought objectives (uses and gratifications theory), emotional factors (affective disposition) and sociological factors are the most prominent to consider in the Moroccan market.

On a scientific level, this work attempts to open a new research path, by establishing a link between the digital viewing trends and viewers' loyalty, through multiplatform strategies. Furthermore, it sheds the light on media use models and their role in predicting viewers' choices of contents. Most importantly, this work helps to identify the most useful variables to consider when conducting a quantitative study of viewership behaviors in general and viewers loyalty in particular. One of the most interesting variables revealed is content, on which there has been little discussion in literature.

From a practical point of view, our study seeks a further understanding of Moroccan managers' conception of changes in viewership behaviors and multiplatform strategies in the television industry. It also highlights the innovation mindset in the corporate cultures of Moroccan broadcasters.

As any scientific research work remains incomplete, this work could have been more enriching, taking into account the following points:

- Enlarging the sample to reach more broadcasters outside of Morocco, namely in the MENA region and in Europe, in order to be able make a cross national comparison ;

- Reach pay TV broadcasters to study their particularities, especially with their different business models ;

- Introducing more variables in the interview such as the living area (urban / rural). 
Finally, this study has gone towards enhancing our understanding the recent trends in television industry from a managerial point of view. This topic is one of the best choices for a researcher combining marketing with media studies in his works. Furthermore, media industries, especially television industry, are still evolving and leading us to future research perspectives, by deepening studies, expanding samples and introducing other variables related to content, convergence, or second screens [13].

\section{REFERENCES}

[1] J. E. Crawford, Cutting the Cord: A Marketing Case: An Examination of Changing TV Viewership. Atlantic Marketing Journal, Vol. 5, No 2 , 136-150, 2016

[2] L. Perticoz \& C. Dessinges, Du téléspectateur au télé-visionneur : Les séries télévisées face aux mutations des consommations audiovisuelles, Études de communication, $n^{\circ} 44,115-130,2015$.

[3] T. Leaver, 'Watching Battlestar Galactica in Australia and the Tyranny of Digital Distance', Media International Australia 126: 145-54, 2008

[4] M. Krcmar, D. Ewoldsen, A. Koerner, Communication Science Theory and Research, New York: Routledge, 2016.

[5] M. Medina, M. Herrero \& E. Guerrero, Audience behaviour and multiplatform strategies: the path towards connected TV in Spain. Austral Comunicacion, vol.4, $n^{\circ} 1,2015$.

[6] L, Hecht, Developments in Television Viewership, CUNY Academic Works, 2016

[7] M. Pittman, \& K. Sheehan, Sprinting a media marathon: Uses and gratifications of binge-watching television through Netflix, First Monday.

[Online]. http://journals.uic.edu/ojs/index.php/fm/article/view/6138/4999, 2015

[8] M. Jenner, Binge-watching: Video-on-demand, quality-TV and mainstreaming fandom, International Journal of Cultural Studies, 2015.

[9] C, Tryon, TV got better: Netflix's original programming strategies and binge viewing, Media Industry Journal, 2, 104-116, 2015.

[10] L. Mikos, Digital Media Platforms and the Use of TV Content: Binge Watching and Video-on-Demand in Germany, Media and Communication, vol.4, $\mathrm{n}^{\circ} 3,154-161,2016$.

[11] F. Bardhi, A. Rohm \& F. Sultan, Tuning in and tuning out: media multitasking among young consumers, Journal of Consumer Behaviour, 9 (4):316-332, 2010.

[12] R. C. Nee \& D. M. Dozier, Second screen effects. Convergence: The International Journal of Research into New Media Technologies, 23(2), 214-226, DOI: 10.1177/1354856515592510, 2016.

[13] A. Dadouh, \& A. Aomari, Moroccan youth's consumption of TV programs during Ramadan and changes of the digital era, European Journal of Management and Marketing Studies, Vol. 4, No 3, 35-51, 2019.

[14] G. Gudorf, With TV, simple is better than smart. IBE: International Broadcast Engineer: 18-20, 2012.

[15] J, Sasseen, K. Olmstead \& A. Mitchell, The state of the news media 2013. The Pew Research Center's Project for Excellence in Journalism, 2013.

[16] E. See-To, S, Papagiannidis, \& V. Cho, User experience on mobile video appreciation: How to engross users and to enhance their enjoyment in watching mobile video clips, Technological Forecasting \& Social Change, 79, 1484-1494, 2012.

[17] S. Winter, N. C. Krämer, B. Benninghoff \& C. Gallus, Shared Entertainment, Shared Opinions: The Influence of Social TV Comments on the Evaluation of Talent Shows, Journal of Broadcasting \& Electronic Media, 62:1, 21-37, DOI: 10.1080/08838151.2017.1402903, 2018.

[18] M. Guo, How Television Viewers Use Social Media to Engage with Programming: The Social Engagement Scale Development and Validation, Journal of Broadcasting \& Electronic Media, 62:2, 195214, DOI: 10.1080/08838151.2018.1451856, 2018.

[19] L. Küng, Innovation, Technology and Organisational Change Legacy Media's Big Challenges, in Stursul T. \& Krumsvik A. H., Media Innovations, a multidisciplinary study of change, Nordicom. University of Gothenburg, 9-12, 2015.

[20] S. Baumann, \& T. C. Hasenpusch, Multi-Platform Television and Business Models: A Babylonian Clutter of Definitions and Concepts, Westminster Papers in Communication and Culture, 11(1), 85-102, DOI: $10.16997 /$ wpcc. $219,2016$.
[21] J. A. García-Avilés, Roles of audience participation in multiplatform television: From fans and consumers, to collaborators and activists. Journal of Audience and Reception Studies. Vol. 9, no 2, 429-447. 2012

[22] A. Fleury, J. S. Pedersen, M. Baunstrup \& L. B. Larsen, Interactive TV Interaction and Control in Second-screen TV Consumption. Presented at the European Interactive TV Conference (EuroITV) (104-107). Fraunhofer Institute for Open Communication Systems, FOKUS, 2012.

[23] Mawenzi Partners, Le regard \#6 - Social TV : les réseaux sociaux sontils en passe de sauver la télévision ?, Mawenzi Partners, 2013.

[24] D. J. Sabavala \& D. G. Morrison, A model of TV show loyalty, Journal of Advertising Research, 17(6), 35-43, 1977.

[25] J. Cohen, Television Viewing Preferences: Programs, Schedules, and the Structure of Viewing Choices Made by Israeli Adults, Journal of Broadcasting \& Electronic Media, 46:2, 204-221. DOI 10.1207/s15506878jobem4602_3, 2002.

[26] L. S. Su \& S. C. Chen, Exploring the Typology and Impacts of Audience Gratifications Gained from TV-Smartphone Multitasking. International Journal of Human-Computer Interaction, DOI 10.1080/10447318.2019.1683312, 2019.

[27] E. Katz, M. Gurevitch, \& H. Haas, On the use of the mass media for important things. American Sociological Review, 38, 164-181. 1973.

[28] S. Finn, Origins of media exposure. Communication Research, 24, 507-529, 1997.

[29] J. B. Weaver, Exploring the links between personality and media preferences, Personality and Individual Differences, 12, 1293-1299, 1991.

[30] D. Zillmann, \& J. Cantor, Affective responses to the emotions of a protagonist, Journal of Experimental Social Psychology, 13(2), 155165, 1977.

[31] A. A. Raney, The psychology of disposition-based theories of media enjoyment, In J. Bryant \& P. Vorderer (dir), Psychology of entertainment, 137-150, Mahwah, NJ: Erlbaum, 2006.

[32] S. Knobloch, Mood adjustment via mass communication, Journal of Communication, 53, 233-250, 2003.

[33] V. C. Strasburger, B. J. Wilson, \& A. B. Jordan, Children, adolescents, and the media, 3rd ed. Thousand Oaks, CA: Sage, 2009.

[34] Z. Papacharissi \& A. M. Rubin, Predictors of Internet Use, Journal of Broadcasting \& Electronic Media, 44, 175-196. http://dx.doi.org/10.1207/s15506878jobem4402_2, 2000.

[35] R. Saxena, Contextual age: A better indicator of aging than chronological age, International Journal of Social Sciences and Management Research, vol. $2 \mathrm{n}^{\circ} 1,2016$.

[36] D. Gauntlett, \& A. Hill, TV Living: television, culture and everyday life, British Film Institute, London and New York, 1999.

[37] B. Bondad-Brown, R. Rice \& K, Pearce, Influences on TV Viewing and Online User-shared Video Use: Demographics, Generations, Contextual Age, Media Use, Motivations, and Audience Activity, Journal of Broadcasting \& Electronic Media, 56:4, 471-493, DOI 10.1080/08838151.2012.732139, 2012

[38] N. K. Denzin, \& Y. S. Lincoln, Paradigms and perspectives in contention, The Sage handbook of qualitative research, 183-190, 2005

[39] S. B. Merriam, Qualitative Research and Case Study Applications in Education, Revised and Expanded from "Case Study Research in Education", Jossey-Bass Publishers, San Francisco, CA, 1998.

[40] A. D. Robert \& A. Bouillaguet, L'analyse de contenu, Paris : Presses Universitaires de France, 2007.

A. Dadouh, born in Meknes - Morocco. He has a Master's Degree in Marketing from Mohammed V University of Rabat.

Currently, he is a Marketing Manager at the Moroccan News Agency, with a total experience of 7 years in media and travel industries. In parallel, he is a PhD student at Mohammed V University of Rabat. His main research interests are marketing communication and media.

He also participated as a speaker at the $17^{\text {th }}$ International Marketing Trends Conference, which was held in Paris from $18^{\text {th }}$ to $20^{\text {th }}$ January 2018 Furthermore, M. DADOUH has published a paper on under the theme "Moroccan Youth's Consumption of TV Programs During Ramadan and Changes of the Digital Era", with Prof. AOMARI from Mohammed V University. 